\title{
ON THE SPECTRAL CHARACTER OF TOEPLITZ OPERATORS ON MULTIPLY CONNECTED DOMAINS
}

\author{
KEVIN F. CLANCEY
}

\begin{abstract}
An explicit resolvent formula is given for selfadjoint Toeplitz operators acting on the least harmonic majorant Hardy spaces of a multiply connected planar domain. This formula is obtained by using theta functions associated with the double of the domain. Several consequences concerning the spectral resolutions of selfadjoint Toeplitz operators are deduced.
\end{abstract}

A selfadjoint operator acting on a Hilbert space admits a diagonalization on a direct integral space with respect to a scalar spectral measure on the real line. This scalar spectral measure can be decomposed relative to Lebesgue measure into its atomic, absolutely continuous and singular continuous components. In many concrete selfadjoint problems there is no singular continuous component. This paper is concerned with the conjecture that for selfadjoint Toeplitz operators on multiply connected planar domains the singular continuous component is absent.

In the classical case where the domain is the unit disc, Rosenblum $[10,11]$ (see also the announcement by Ismagilov [7]) has shown that a selfadjoint Toeplitz operator which is not a multiple of the identity is absolutely continuous. Actually, the work of these authors goes much further in that they provide a concrete diagonalization complete with multiplicity function and implementing unitary.

More recently, Pincus and Xia [9] have established that on a multiply connected domain, selfadjoint Toeplitz operators cannot be purely singular continuous. In fact, Pincus and Xia establish that the only way a selfadjoint Toeplitz operator on a multiply connected domain can be purely singular is when its symbol is constant on each component of the boundary. In this latter case, the spectrum and spectral multiplicity function has been described explicitly in Clancey [4]. Further, Pincus and Xia determine the spectral multiplicity of the absolutely continuous component and give bounds on the dimension of the eigenspaces in terms of the connectivity of the domain.

Here it will be shown that on a doubly connected domain a selfadjoint Toeplitz operator cannot have a singular continuous component. Further, on

Received by the editors February 17, 1989.

1980 Mathematics Subject Classification (1985 Revision). Primary 47B35; Secondary 47B25, $30 \mathrm{D} 55$. 
an arbitrary multiply connected domain, a selfadjoint Toeplitz operator whose symbol is either analytic or a simple function cannot have a singular continuous component. Although this is strong evidence for the conjecture, the general case remains open. One important feature of the approach here is that an explicit formula is obtained for the resolvent in terms of Riemann theta functions.

It is time to be more specific. Let $D$ denote a $g$-holed planar domain whose positively oriented boundary $\partial D$ is the union of the disjoint simple closed analytic curves $b_{0}, b_{1}, \ldots, b_{g}$, where $b_{0}$ bounds the unbounded component of the complement of $D$ and $b_{1}, \ldots, b_{g}$ bound the $g$ holes. Fix $a$ in $D$. Let $m_{a}$ denote harmonic measure on $\partial D$ based at $a$. The closure $H^{2}\left(m_{a}\right)$ in $L^{2}\left(m_{a}\right)$ of the functions holomorphic on the closure of $D$ is called the least harmonic majorant Hardy space of $D$ based at $a$. The orthogonal projection of $L^{2}\left(m_{a}\right)$ onto $H^{2}\left(m_{a}\right)$ will be denoted by $P$. Given $\phi$ in $L^{\infty}\left(m_{a}\right)$ one defines the Toeplitz operator $T_{\phi}$ on $H^{2}\left(m_{a}\right)$ by $T_{\phi} f=P \phi f, f$ in $H^{2}\left(m_{a}\right)$. The map $\phi \rightarrow T_{\phi}$ is a *-linear isometry from $L^{\infty}\left(m_{a}\right)$ into the $C^{*}$ algebra of bounded linear operators on $H^{2}\left(m_{a}\right)$. In particular, the Toeplitz operator $T_{\phi}$ is selfadjoint if and only if the symbol $\phi$ is real-valued. An excellent reference containing the elementary properties of Toeplitz operators on multiply connected domains is Abrahamse [1].

The method used here will be to exploit the function theory of the Schottky double of the planar domain. Recall that the double of a $g$-holed planar domain $D$ as described above is a compact Riemann surface $X$ of genus $g$. This surface $X$ is obtained by gluing a second copy $D^{\prime}$ of $D$ to $D \cup \partial D$ along $\partial D$. The conformal structure on $Y=D \cup \partial D$ is the usual conformal structure of $Y$ considered as a bordered Riemann surface and the structure on $D^{\prime}$ is the conjugate of that on $D$. Thus the mapping $J: X \rightarrow X$ interchanging points in $D$ with their twins in $D^{\prime}$ is an antiholomorphic involution on the double $X$. Let $G=G(z, a)$ be the Green's function for $D$ with pole at $z=a$. The meromorphic differential $-(\pi i)^{-1} \partial G d z\left(\partial=2^{-1}\left[\partial_{x}-i \partial_{y}\right]\right)$ on the closure reflects to an element in the space $\mathscr{K}^{1}(X)$ of meromorphic differentials on $X$, which is symmetric in the sense that $J^{*} d w_{a}=\overline{d w}_{a}$ and such that the restriction of $d w_{a}$ to $\partial D$ is precisely $d m_{a}$. The zero divisor of $d w_{a}$ (elements in the divisor group $\operatorname{Div}(X)$ of $X$ will be written additively) has the form $\mathscr{D}_{a}+J \mathscr{D}_{a}$, where $\mathscr{D}_{a}=p_{1}+\cdots+p_{g}$ is the critical Green's divisor (this means $p_{1}, \ldots, p_{g}$ are the points in $D$ where $\partial G$ is zero). Let $K^{2}\left(m_{a}\right)$ be the closure in $L^{2}\left(m_{a}\right)$ of the meromorphic functions $f$ on $J Y$ such that the divisor $(f)$ of $f$ satisfies $(f) \geq J a-J \mathscr{D}_{a}$. The orthogonal decomposition

$$
L^{2}\left(m_{a}\right)=H^{2}\left(m_{a}\right) \oplus K^{2}\left(m_{a}\right)
$$

allows one to analyze Toeplitz operators in terms of the function theory on the double. It will be important that $H^{2}\left(m_{a}\right)$ is a reproducing kernel Hilbert space. This means that given $z$ in $D$, there is an element $k_{z}$ in $H^{2}\left(m_{a}\right)$ satisfying 
$f(z)=\left\langle f, k_{z}\right\rangle$, for any $f$ in $H^{2}\left(m_{a}\right)$, where $\langle\cdot, \cdot\rangle$ denotes the inner product in $H^{2}\left(m_{a}\right)$. In fact, the key method used here is to solve "explicitly" the equations $\left(T_{\phi}-\lambda\right) x=k_{z}$ using the techniques of Hilbert barrier problems on Riemann surfaces as developed in Zverovich [12].

As usual, the theta function associated with the Riemann surface $X$ can be used effectively in the situation here. It is necessary to mark the double by choosing a canonical homology basis $a_{1}, \ldots, a_{g}: b_{1}, \ldots, b_{g}$ on $X$. This is conveniently accomplished by completing the $B$-cycles $b_{1}, \ldots, b_{g}$ to such a homology basis as follows. Fix $p_{0}$ in $b_{0}$. Let $\alpha_{j}$ be a crosscut in $D$ from $p_{0}$ to $b_{j}$ and let $a_{j}=\alpha_{j} \cup-J \alpha_{j}, j=1, \ldots, g$, form the $A$-cycles. Let $d w_{1}, \ldots, d w_{g}$ be a basis for the space $\Omega(X)$ of holomorphic one forms dual to the above canonical homology basis. This basis of $\Omega(X)$ can be described explicitly as follows. Let $\omega_{j}=\omega_{j}(z)$ be the harmonic measure of $b_{j}$ and reflect the holomorphic differential $d w_{j}=\partial w_{j} d z$ from $Y$ to a holomorphic differential still denoted $d w_{j}$ on $X, j=1, \ldots, g$. Let $d \vec{w}$ be the vector of holomorphic forms $d \vec{w}=\left(d w_{1}, \ldots, d w_{g}\right)^{t}$ (the " $t$ " denotes transpose). The homology basis and the dual basis of $\Omega(X)$ have the requisite property that the $g \times 2 g$ Riemann period matrix has the form

$$
\left[\int_{a_{1}} d \vec{w}, \ldots, \int_{a_{g}} d \vec{w}: \int_{b_{1}} d \vec{w}, \ldots, \int_{b_{g}} d \vec{w}\right]=\left[I_{g}: \tau\right],
$$

where $I_{g}$ is the $g \times g$ identity matrix and the $B$-period matrix $\tau$ is of the form $\tau=i P$ with $P$ a positive definite real symmetric matrix. The complex torus $\operatorname{Jac}(X)=\mathbf{C}^{g} /\left(\mathbf{Z}^{g}+\tau \mathbf{Z}^{g}\right)$ is called the Jacobian variety of the marked double. The double $X$ is mapped in a biholomorphic manner into $\operatorname{Jac}(X)$ by the Abel-Jacobi map $\zeta_{0}: X \rightarrow \operatorname{Jac}(X)$ defined by

$$
\zeta_{0}(p)=\int_{p_{0}}^{p} d \vec{w} \bmod \left(\mathbf{Z}^{g}+\tau \mathbf{Z}^{g}\right),
$$

where the base point $p_{0}$ will always be chosen in $b_{0}$. The Abel-Jacobi map can be combined with the theory of the theta function to completely analyze the function theory of $X$. Recall that given a symmetric $g \times g$ complex matrix $\tau$ whose imaginary part is positive definite one defines the theta function as the entire function defined on $\mathbf{C}^{g}$ by

$$
\theta(z)=\theta(z, \tau)=\sum_{n \in \mathbf{Z}^{g}} \exp 2 \pi i\left(\frac{1}{2} n^{t} \tau n+n^{t} z\right) .
$$

The main result of this paper is

Theorem 1. Let $\phi$ be a real valued element in $L^{\infty}\left(m_{a}\right)$ and $T_{\phi}$ the corresponding Toeplitz operator on $H^{2}\left(m_{a}\right)$. For $z$ in $D$ let $k_{z}$ denote the reproducing 
kernel on $H^{2}\left(m_{a}\right)$. For any complex $\lambda$ satisfying $\operatorname{Im} \lambda \neq 0$, there holds

$$
\begin{aligned}
& \left\langle\left(T_{\phi}-\lambda\right)^{-1} k_{z}, k_{z}\right\rangle \\
& =C(z) \frac{\theta\left(\omega_{z}+t_{a}-(1 / 2 \pi i) \int_{\partial D} \log (\phi-\lambda) d \vec{w}\right)}{\theta\left(t_{a}-(1 / 2 \pi i) \int_{\partial D} \log (\phi-\lambda) d \vec{w}\right)} e^{-\int \log (\phi-\lambda) d m_{z}},
\end{aligned}
$$

where $t_{a}$ is a constant and $\omega_{z}, C(z)$ are constants depending only on $z$.

The resolvent representation in the above theorem should be compared with the result appearing as equation $\left(2.5^{\prime}\right)$ in [11]. In the case of the unit disc the resolvent formula is as above without the ratio of theta functions factor. It is the denominator of this factor which permits the presence of singular spectra in the case of a multiply connected domain.

The above theorem will be used to rule out the presence of singular continuous spectra in case the domain is doubly connected or the symbol $\phi$ is analytic or a simple function. Further, this representation of the resolvent yields explicit formulae for the absolutely continuous component of the spectral measure. It should be noted that the vector constants $t_{a}, \omega_{z}$ and the real constant $C(z)$ all have explicit form.

The remainder of this paper is organized as follows. $\S 1$ contains preliminaries on the solution of the relevant Hilbert barrier problem on $X$. The construction of the reproducing kernel for $H^{2}\left(m_{a}\right)$ in terms of the prime form is also given in $\S 1$. The proof of Theorem 1 is given in $\S 2$ and here one will also find the explicit formula for the constant $C(z) . \S 3$ is devoted to spectral analysis of $T_{\phi}$ obtained as consequences of Theorem 1.

\section{Preliminaries}

In this section we will develop the necessary prerequisites from the theory of Riemann surfaces to solve for $\operatorname{Im} \lambda \neq 0$ and for $\phi$ a real valued function in $L^{\infty}\left(m_{a}\right)$ the Toeplitz equation $\left(T_{\phi}-\lambda\right) x=k_{z}$, where $z \rightarrow k_{z}, z$ in $D$, is the reproducing kernel on $H^{2}\left(m_{a}\right)$. To describe the solutions of this equation it will be necessary to recall the techniques for solving the Hilbert barrier problem associated with a divisor along a system of curves on a compact Riemann surface. These barrier problems are discussed in Zverovich [12]. See also the discussion in Clancey [4]. In addition, in order to describe the explicit form of the reproducing kernel $k_{z}$ it will be necessary to recall some standard facts concerning the construction of meromorphic functions and differentials using theta functions. References for this material on theta functions are Fay [6] and Mumford [8]. See also the discussion in Clancey [3].

Any notations and conventions introduced in the introduction remain in effect. The spaces $H^{2}\left(m_{a}\right)$ and $K^{2}\left(m_{a}\right)$ have the following alternate descriptions. Consider the collection $\mathscr{H}^{2}\left(m_{a}\right)$ of functions $g$ analytic on $D$ for which

$$
\|g\|^{2}=\lim _{\varepsilon \downarrow 0}-\frac{1}{2 \pi} \int_{G=\varepsilon}|g|^{2} * d G
$$


is finite. Every element $g$ in $\mathscr{H}^{2}\left(m_{a}\right)$ has a nontangential limit function $g^{+}$ a.e. $m_{a}$ on $\partial D$ and the correspondence $g \rightarrow g^{+}$is a unitary map from $\mathscr{H}^{2}\left(m_{a}\right)$ to $H^{2}\left(m_{a}\right)$. Similarly, let $\mathscr{K}^{2}\left(m_{a}\right)$ denote the meromorphic functions $g$ on $J D$ whose divisors $(g)$ satisfy $(g) \geq J a-\mathscr{D}_{a}$ and for which

$$
\|g\|^{2}=\lim _{\varepsilon \uparrow 0}-\frac{1}{2 \pi} \int_{G=\varepsilon}|g|^{2} * d G
$$

is finite (we have reflected the Green's function into $J D$ ). A function $g$ in $\mathscr{K}^{2}\left(m_{n}\right)$ has a.e. $m_{a}$ a nontangential limit function $g^{-}$on $\partial D$ and the map $g \rightarrow g^{-}$is unitary from $\mathscr{K}^{2}\left(m_{a}\right)$ to $K^{2}\left(m_{a}\right)$. Thus it is clear that for $G$ in $L^{\infty}\left(m_{a}\right)$ and $k$ in $H^{2}\left(m_{a}\right)$ solving the Toeplitz equation $T_{G} x=k$ is equivalent to solving the generalized Hilbert barrier problem:

Find a sectionally meromorphic function $g$ on $X \backslash \partial D$ such that $g$ restricted to $D$ is in $\mathscr{H}^{2}\left(m_{a}\right)$ and $g$ restricted to $J D$ is in $\mathscr{K}^{2}\left(m_{a}\right)$ with

$$
G g^{+}=g^{-}+k \text { a.e. } m_{a} \text { on } \partial D \text {. }
$$

We will describe the usual approach to solving this barrier problem using a multiplicative factorization of the symbol $G$. This necessitates a discussion of the multiplicative Cauchy kernel. Fix a point $b$ on $X$. This point will be taken in $J D$. The notation $d \lambda_{w-b}$ will be used for the unique element in the space $\mathscr{M}^{1}(X)$ of meromorphic differentials on $X$ having zero periods along the cycles $a_{1} \cdots a_{g}$, simple poles at $w$ and $b$, with $\operatorname{Res}_{z=w} d \lambda_{w-b}=1$. The multiplicative Cauchy kernel $A(z, w) d z=d \lambda_{w-b}(z)$ has the following properties.

1. For fixed $w \neq b, A(z, w) d z$ is in $\mathscr{M}^{1}(X)$ with divisor $(A(z, w)) \geq$ $-w-b$ and expansion in local coordinates near $w$ in the form

$$
A(z, w) d z=d z /(z-w)+d h(z, w),
$$

where $h(z, w)$ is holomorphic at $z=w$.

2. For $z$ fixed, $A(z, w) d z$ is an abelian integral in its dependence on $w$ which satisfies

$$
\int_{a_{j}} d_{w} A(z, w) d z=0, \quad \int_{b_{j}} d_{w} A(z, w) d z=2 \pi i d w_{j}(z), \quad j=1, \ldots, g
$$

where again $d w_{1}, \ldots, d w_{g}$ is our basis for $\Omega(X)$.

Suppose that $F$ is a bounded measurable function on $D$ (measurability here means with respect to either arc length measure or the equivalent measure $m_{a}$ ). Consider the function

$$
H(p)=\exp \left(\frac{1}{2 \pi i} \int_{\partial D} F(t) A(t, p) d t\right) .
$$

The function $H$ is a multiple-valued holomorphic function on $X \backslash \partial D$ with the following continuation properties: Suppose that $\gamma$ is a closed curve in $D$ (respectively $J D$ ) homologous to $n_{1} b_{1}+\cdots+n_{g} b_{g}$ (where $n_{1}, \ldots, n_{g}$ are 
integers) in $Y$ (respectively $J Y$ ). Then continuation of a germ $H_{0}$ of $H$ around $\gamma$ produces the germ $\chi_{F}(\gamma) H_{0}$, where $\chi_{F}$ is the homology character

$$
\chi_{F}\left(b_{j}\right)=\exp \left(\int_{\partial D} F d w_{j}\right), \quad j=1, \ldots, g .
$$

The theta function can be conveniently used to remove this multiplicative behavior of the function $H$ defined in (1.1). We recall that the theta function is quasi-periodic in the sense that

$$
\theta(z+m+\tau n, \tau)=\exp 2 \pi i\left(-\frac{1}{2} n^{t} \tau n-n^{t} z\right) \theta(z, \tau),
$$

for $z$ in $\mathbf{C}^{g}$ and $m, n$ in $\mathbf{Z}^{g}$. Consequently, for $c, d$ in $\mathbf{C}^{g}$, the function defined for $p$ in $X$ by

$$
f(p)=\frac{\theta\left(\int_{p_{0}}^{p} d \vec{w}-c\right)}{\theta\left(\int_{p_{0}}^{p} d \vec{w}-d\right)}
$$

is a multiplicative meromorphic function belonging to the character

$$
\chi\left(a_{j}\right)=1 ; \quad \chi\left(b_{j}\right)=\exp 2 \pi i\left(c_{j}-d_{j}\right), \quad j=1, \ldots, g .
$$

Thus if one chooses $c, d$ in $\mathbf{C}^{g}$ such that

$$
d-c=\frac{1}{2 \pi i} \int_{\partial D} F d \vec{w}
$$

and such that the function $f$ defined by (1.2) is not identically zero or infinite (such a choice of $c, d$ is always possible), then the function $f H$ is a single-valued meromorphic function on $X \backslash \partial D$. The function $f H$ also has the property that a.e. on $\partial D$ the nontangential limits $(f H)^{+},(f H)^{-}$exist and satisfy the barrier problem $(f H)^{+}=e^{F}(f H)^{-}$.

Remarks. (i) Even after it is arranged that the function $f$ has no zeros or poles in a neighborhood of $\partial D$, it is not obvious that the sectionally meromorphic function $g=f H$ satisfies

$$
\lim _{\varepsilon \rightarrow 0} \int_{G= \pm \varepsilon}|g|^{2} * d G<\infty
$$

When the function $F$ is sufficiently smooth (say Hölder continuous), then $g$ will be continuous up to the boundary and (1.3) will hold. Fortunately, in the arguments needed here we will be able to limit the use of functions of the form (1.1) to the case where $F$ is smooth.

(ii) It is clear that when $F=\log \phi$, then with $\phi^{+}=(f H)^{+}$and $\phi^{-}=$ $\left[(f H)^{-}\right]^{-1}$, at least formally, we have the "Wiener-Hopf" factorization $\phi=$ $\phi^{+} \phi^{-}$.

We now turn to a brief development of the Klein prime form on $X$. This form is the basic object for the explicit representation of meromorphic functions and differentials on $X$. 
The prime form construction is based on the existence of a nonsingular odd half-period. This half-period is a point $[e]=\frac{1}{2} \mu_{0}+\frac{1}{2} \tau \nu_{0}$ in $\operatorname{Jac}(X)$ where $\mu_{0}, \nu_{0}$ are in $\mathbf{Z}^{g} / 2 \mathbf{Z}^{g}$ such that $\mu_{0}^{t} \nu_{0}$ is odd (so that $\theta([e])=0$ ) and $\nabla \theta$ is not zero at $[e]$. The existence of a nonsingular odd half-period is established in Mumford [8]. We will let $\theta[e]$ denote the theta function with characteristics $a=\frac{1}{2} \nu_{0}, b=\frac{1}{2} \mu_{0}$. Thus

$$
\theta[e](z, \tau)=\theta\left[\begin{array}{l}
a \\
b
\end{array}\right](z, \tau)=\exp \left\{2 \pi i\left(\frac{1}{2} a^{t} \tau a+a^{t}(z+b)\right)\right\} \theta(z+b+\tau a) .
$$

We remark that $\theta[e](\bar{z})=\theta \overline{[e](z)}$. The function defined on $X \times X$ by

$$
F(z, w)=\theta[e]\left(\int_{z}^{w} d \vec{w}\right)
$$

is a multiple-valued holomorphic function with the following property: For $z$ (respectively, $w$ ) fixed in $X$, the multiple-valued holomorphic function $F(z, \cdot)$ (respectively, $F(\cdot, w)$ ) has zero divisor $p_{1}+\cdots+p_{q-1}+z$ (respectively, $p_{1}+$ $\left.\cdots+p_{g-1}+w\right)$ where the divisor $\mathscr{E}=p_{1}+\cdots+p_{g-1}$ is independent of $z$ (respectively, $w$ ) and satisfies $\zeta_{0}(\mathscr{E})+\Delta_{0}=[e]$. The constant $\Delta_{0}$ appearing in this last equation is Riemann's constant based at $p_{0}$. This important constant has the property that when, for $c$ fixed in $\mathbf{C}^{g}$, the multiple-valued holomorphic function

$$
h(p)=\theta\left(\int_{p_{0}}^{p} d \vec{w}-c\right)
$$

does not vanish identically, then its zero divisor $p_{1}+\cdots+p_{g}$ satisfies

$$
\zeta_{0}\left(p_{1}+\cdots+p_{g}\right)+\Delta_{0}=[c] \text {. }
$$

Thus it is possible to characterize the divisor, for example, of a multiplicative meromorphic function of the form (1.2).

The explicit form of the Riemann constant is

$$
\Delta_{0}=\left[\sum_{k=1}^{g}\left(\int_{a_{k}} \vec{w}_{0}(p) d w_{k}-\frac{1}{2} \tau_{k k} e_{k}\right)\right],
$$

where $\vec{w}_{0}(p)=\int_{p_{0}}^{p} d \vec{w}$ and $e_{1}, \ldots, e_{g}$ is the standard basis in $\mathbf{C}^{g}$. We immediately observe that the constant $\Delta_{0}$ satisfies $J \Delta_{0}=\Delta_{0}$, where for [z] in $\operatorname{Jac}(X)$ one defines the involution $J[z]=-[\bar{z}]$. This involution also has the property that $\zeta_{0} J=J \zeta_{0}$, where we emphasize that this requires the base point $p_{0}$ of the Abel-Jacobi map to be in $\partial D$. Further, $-2 \Delta_{0}=K_{X}$, where $K_{X}$ is the value of the Abel-Jacobi map (based at $p_{0}$ ) on the canonical class. This means $K_{X}=\zeta_{0}((d w))$, where $d w$ is any meromorphic differential on $X$.

Since the divisor $\mathscr{E}$ satisfies $\zeta_{0}(2 \mathscr{E})=-2 \Delta_{0}$, then there must be an element $d w_{\mathscr{E}}$ in $\Omega(X)$ with $\left(d w_{\mathscr{E}}\right)=2 \mathscr{E}$. Indeed, this holomorphic one-form is given by

$$
d w_{\mathscr{E}}=\sum_{j=1}^{g} \frac{d \theta[e]}{d z_{j}}(0) d w_{j}
$$


The (class of the) holomorphic line bundle $L_{\mathscr{E}}$ over $X$ determined by the divisor class of $\mathscr{E}$ has the property that $L_{\mathscr{E}} \otimes L_{\mathscr{E}}$ is equivalent to the canonical bundle. Choose a holomorphic section $h_{\mathscr{E}}$ of $L_{\mathscr{E}}$ such that $h_{\mathscr{E}}^{2}=d w_{\mathscr{E}}$.

The prime form $E$ is defined by

$$
E(z, w)=\frac{\theta[e]\left[\int_{z}^{w} d \vec{w}\right]}{h_{\mathscr{E}}(z) h_{\mathscr{E}}(w)} .
$$

This form $E$ can be considered as a holomorphic form of weight $-\frac{1}{2},-\frac{1}{2}$ on $X^{\prime} \times X^{\prime}$, where $X^{\prime}$ is the universal cover of $X$. Fix $w_{0}$ in $X$. Then $E\left(z, w_{0}\right)$ is a multiple-valued holomorphic differential of weight $-\frac{1}{2}$ in the variable. The multiple-valued nature of $E\left(z, w_{0}\right)$ (which arises from the function $\theta[e]$ appearing in the form $\left.E\left(z, w_{0}\right)\right)$ can be described as follows. Fix coordinate charts at $z_{0}$ and $w_{0}$. Beginning and ending at $z_{0}$, continue $E\left(z, w_{0}\right)$ along a cycle which is homologous to $\Sigma\left(n_{j} a_{j}+m_{j} b_{j}\right)$. When $E\left(z, w_{0}\right)$ is computed near $z_{0}$ in the same coordinate charts, then this continuation produces a change in $E\left(z, w_{0}\right)$ by the multiplicative factor

$$
\exp \pi i\left(\nu_{0} m-\mu_{0} n-m \tau \mu_{0}\right) \exp \left(2 \pi i m \int_{z}^{w_{0}} d \vec{w}\right),
$$

where $n=\left(n_{1}, \ldots, n_{g}\right)$ and $m=\left(m_{1}, \ldots, m_{g}\right)$. The most important feature of $E\left(z, w_{0}\right)$ is that its divisor is well defined and equals $w_{0}$.

The representing measure $m_{a}$ has the property that on $\partial D$

$$
d m_{a}=d \lambda_{a-J a} / 2 \pi i \text {. }
$$

For simplicity of notation we will write $d w_{a}$ for the meromorphic differential $d \lambda_{a-J a} / 2 \pi i$ which restricts to $d m_{a}$ on $\partial D$. The divisor of the differential has the form $\mathscr{D}_{a}-a+J\left(\mathscr{D}_{a}-a\right)$ where $\mathscr{D}_{a}=z_{1}(a)+\cdots+z_{g}(a)$ is the critical Green's divisor consisting of those points in $D$ (counting multiplicity) where $d w_{a} / d z$ vanishes. It turns out that the point

$$
t_{a}=\zeta_{0}\left(\mathscr{D}_{a}\right)-\zeta_{0}(a)+\Delta_{0}
$$

is real point $\left(t_{a} \in \mathbf{R}^{g} / \mathbf{Z}^{g}\right)$ in $\operatorname{Jac}(X)$. The function $\theta$ does not vanish on the real torus $\mathbf{T}_{0}=\mathbf{R}^{g} / \mathbf{Z}^{g}$ in $\operatorname{Jac}(X)$. Moreover, $t_{a}$ is a point in $\mathbf{T}_{0}$ satisfying

$$
\nabla \log \left(\theta(x) / \theta\left(x+\omega_{a}\right)\right)=0,
$$

where $\omega_{a}=\left(\omega_{1}(a), \ldots, \omega_{g}(a)\right)^{t}$ is the vector with $j$ th component the value $\omega_{j}(a)$ of harmonic measure of $b_{j}(j=1, \ldots, g)$ based at $a$. It should be remarked that $\theta$ is $\mathbf{Z}^{g}$ periodic, consequently, it makes sense to speak of solutions of (1.4) in $\mathbf{T}_{0}$.

We want to describe the differentials $d w_{a}$ in terms of the theta function and the prime form. This representation is given in Fay [6]. See also [3], where the normalizations are consistent with those in this paper. The following representation holds:

$$
d w_{a}(p)=\frac{1}{2 \pi i} \frac{E(a, J a)}{E(a, p) E(p, J a)} \frac{\theta\left(\int_{J a}^{p} d \vec{w}+t_{a}\right) \theta\left(\int_{a}^{p} d \vec{w}-t_{a}\right)}{\theta\left(t_{a}+\omega_{a}\right) \theta\left(t_{a}\right)} .
$$


In (1.5) and from now on $t_{a}$ will denote a fixed point in $\mathbf{R}^{g}$ representing $t_{a}$ considered as an element in $\operatorname{Jac}(X)$.

Note that the reproducing kernel $k_{z}, z$ in $D$, has the form

$$
k_{z}=P\left(d w_{z} / d w_{a}\right),
$$

where $P$ is the orthogonal projection onto $H^{2}\left(m_{a}\right)$. This presentation of $k_{z}$ is not adequate for our purposes. The following representation of the reproducing kernel appears as Proposition 6.15 in Fay [6].

Proposition 1. For $z$ in $D$, the function

$$
k_{z}(p)=\frac{\theta\left(\int_{J z}^{p} d \vec{w}+t_{a}\right) \theta\left(\int_{J a}^{a} d \vec{w}+t_{a}\right)}{\theta\left(\int_{J z}^{a} d \vec{w}+t_{a}\right) \theta\left(\int_{J a}^{p} d \vec{w}+t_{a}\right)} \frac{E(J z, a) E(p, J a)}{E(J a, a) E(p, J z)}
$$

is an element in $H^{2}\left(m_{a}\right)$ with the property that $f(z)=\left\langle f, k_{z}\right\rangle$, for every $f$ in $H^{2}\left(m_{a}\right)$.

Proof. It is easily verified that the meromorphic function defined on $X$ in (1.6) has (for $z \neq a$ ) the divisor

$$
\left(k_{z}\right)=Q_{1}(z)+\cdots+Q_{g}(z)+J a-J \mathscr{D}_{a}-J z,
$$

where

$$
\zeta_{0}\left(Q_{1}(z)+\cdots+Q_{g}(z)\right)=J z+J t_{a}+\Delta_{0} .
$$

In particular, $k_{z}$ is holomorphic and belongs to $H^{2}\left(m_{a}\right)$. On $\partial D$ it can be checked that

$$
\bar{k}_{z}(p)=\frac{\theta\left(\int_{z}^{p} d \vec{w}+t_{a}\right) \theta\left(\int_{a}^{J a} d \vec{w}+t_{a}\right)}{\theta\left(\int_{z}^{J a} d \vec{w}+t_{a}\right) \theta\left(\int_{a}^{p} d \vec{w}+t_{a}\right)} \frac{E(z, J a) E(p, a)}{E(a, J a) E(p, z)} .
$$

Further, it can be verified that the meromorphic differential $\bar{k}_{z}(p) d w_{a}(p)$ has only a simple pole in $\bar{D}$ at $p=z$ with residue $(2 \pi i)^{-1}$. Consequently, for any $f$ in $H^{2}\left(m_{a}\right)$,

$$
f(z)=\int_{\partial D} f \bar{k}_{z} d w_{a}=\left\langle f, k_{z}\right\rangle
$$

This completes the proof of the proposition.

\section{Proof of Theorem 1}

Let $\phi$ be a real-valued element in $L^{\infty}\left(m_{a}\right)$. For $\operatorname{Im} \lambda \neq 0$, we will let $\log (\phi-\lambda)$ denote the principal determination of the logarithm of the function $\phi-\lambda$, where $-\pi<\operatorname{Im} \log z \leq \pi$.

Assume first that the symbol $\phi$ is smooth (for example, it is sufficient to assume that $\phi$ is Hölder continuous of order $0<\alpha<1$ as a function of arclength on $\partial D$ ).

Fix $\lambda$ with $\operatorname{Im} \lambda \neq 0$. Let $H$ be the multiple-valued holomorphic function defined on $X \backslash \partial D$ by

$$
H(p)=\exp \left(\frac{-1}{2 \pi i} \int_{\partial D} \log (\phi-\lambda) A(t, p) d t\right),
$$


where $A(t, p) d t$ is the multiplicative Cauchy kernel introduced above.

We are going to multiply $H$ by the multiplicative function

$$
f_{z}(p)=\frac{\theta\left(\int_{J z}^{p} d \vec{w}+t_{a}-(1 / 2 \pi i) \int_{\partial D} \log (\phi-\lambda) d \vec{w}\right)}{\theta\left(\int_{J a}^{p} d \vec{w}+t_{a}\right)} \frac{E(J z, a) E(p, J a)}{E(J a, a) E(p, J z)},
$$

where $z$ is fixed in $D$.

It may be that for $z$ fixed in $D$ and $\lambda$ nonreal the multiple-valued function

$$
\theta\left(\int_{p_{0}}^{p} d \vec{w}-\zeta_{0}(J z)+t_{a}-\frac{1}{2 \pi i} \int_{\partial D} \log (\phi-\lambda) d \vec{w}\right)
$$

appearing in the numerator in (2.1) vanishes identically on $X$. Nevertheless, for $z$ fixed there is at most a countable set of values of $\lambda$ off the interval $[m, M]$, where $m=\operatorname{essinf} \phi, M=\operatorname{esssup} \phi$, having the property that the function on $X$ defined by (2.2) vanishes identically. One sees this last remark as follows. Note first that, for $\lambda<m$,

$$
t_{a}-\frac{1}{2 \pi i} \int_{\partial D} \log (\phi-\lambda) d \vec{w}
$$

is in $\mathbf{R}^{g}$. Now the function $\theta$ associated with the double of a planar domain does not vanish on $\mathbf{R}^{g}$ (see Fay [6] and also [3]). Thus for $z$ fixed there is at most a countable set of points $\lambda$ off $[m, M]$ where the function defined in (2.2) vanishes at $J z$.

Observe that, under the hypothesis that the function $f_{z}$ is not identically zero, the function $f_{z}$ belongs to the character

$$
\chi\left(a_{j}\right)=1 ; \quad \chi\left(b_{j}\right)=\exp \left(\int_{\partial D} \log (\phi-\lambda) d w_{j}\right), \quad j=1, \ldots, g .
$$

Further, the divisor of $f_{z}$ is given by

$$
\left(f_{z}\right)=\mathscr{D}_{1}-J z+J a-J \mathscr{D}_{a},
$$

where the divisor $\mathscr{D}_{1}$ is of degree $g$ and satisfies

$$
\zeta_{0}\left(\mathscr{D}_{1}\right)+\Delta_{0}=\zeta_{0}(J z)-t_{a}+\left[\frac{1}{2 \pi i} \int_{\partial D} \log (\phi-\lambda) d \vec{w}\right] .
$$

Assuming the hypothesis that $\lambda$ and $z$ are such that $f_{z}$ is nonzero, let $h_{z}=f_{z} H$. This function restricted to $D$ is in $\mathscr{H}^{2}\left(m_{a}\right)$ and satisfies

$$
(\phi-\lambda)\left(h_{z}\right)^{+}=\left(h_{z}\right)^{-} \text {. }
$$

The divisor of the function $h_{z}$ is the same as the divisor of $f_{z}$ and, therefore, $h_{z}$ restricted to $J D$ would be in the space $\mathscr{K}^{2}\left(m_{a}\right)$ except for the pole at $J z$ which must be removed. This pole can be removed by subtracting the appropriate multiple of $k_{z}$. In fact, since both $h_{z}$ and $k_{z}$ have the factor

$$
\frac{E(J z, a) E(p, J a)}{E(J a, a) E(p, J z)}
$$


then it is easy to see that $h_{z}-K(z) k_{z}$ is in $\mathscr{K}^{2}\left(m_{a}\right)$, where

$$
K(z)=\frac{\theta\left(t_{a}-(1 / 2 \pi i) \int_{\partial D} \log (\phi-\lambda) d \vec{w}\right)}{\theta\left(t_{a}\right)} H(J z),
$$

for an appropriate choice of $H(J z)$.

The above discussion shows that

$$
\left(T_{\phi}-\lambda\right) h_{z}=K(z) k_{z} \text {. }
$$

We can now conclude that

$$
\left\langle\left(T_{\phi}-\lambda\right)^{-1} k_{z}, k_{z}\right\rangle=C(z) \frac{\theta\left(\int_{J z}^{z} d \vec{w}+t_{a}-(1 / 2 \pi i) \int_{\partial D} \log (\phi-\lambda) d \vec{w}\right)}{\theta\left(t_{a}-(1 / 2 \pi i) \int_{\partial D} \log (\phi-\lambda) d \vec{w}\right)} \frac{H(z)}{H(J z)}
$$

with

$$
C(z)=\frac{\theta\left(t_{a}\right) \theta\left(\omega_{a}+t_{a}\right) E(J z, a) E(z, J a)}{\theta\left(\int_{J z}^{a} d \vec{w}+t_{a}\right) \theta\left(\int_{J a}^{z} d \vec{w}+t_{a}\right) E(J a, a) E(z, J z)},
$$

a real constant.

Recall that $A(t, p) d t=d \lambda_{w-b}$ for some fixed $b$ in $X$. Now assume this $b$ is chosen as $J z$. It follows that $H(J z)=1$ and

$$
H(z)=\exp \left(-\int_{\partial D} \log (\phi-\lambda) d m_{z}\right) .
$$

Consequently, equation (2.3) can be rewritten in the form

$$
\begin{array}{r}
\left\langle\left(T_{\phi}-\lambda\right)^{-1} k_{z}, k_{z}\right\rangle=C(z) \frac{\theta\left(\omega_{z}+t_{a}-(1 / 2 \pi i) \int_{\partial D} \log (\phi-\lambda) d \vec{w}\right)}{\theta\left(t_{a}-(1 / 2 \pi i) \int_{\partial D} \log (\phi-\lambda) d \vec{w}\right)} \\
\times \exp \left(-\int_{\partial D} \log (\phi-\lambda) d m_{z}\right),
\end{array}
$$

where $C(z)$ is still given by (2.4).

The result in (2.5) was established for $z$ fixed and $\lambda$ satisfying the hypothesis that the function $f_{z}$ was not identically zero. It follows by analytic continuation of both sides of (2.5) that this equation holds with $z$ fixed for all $\lambda$. Finally, the identity $(2.5)$ is extended to an arbitrary $\phi$ in $L^{\infty}\left(m_{a}\right)$ by approximating $\phi$ by a sequence $\phi_{n}$ of Hölder continuous functions which converge pointwise boundedly to $\phi$. The sequence of Toeplitz operators $T_{\phi_{n}}$ converges strongly to $T_{\phi}$. Since (2.5) holds for each of the operators $T_{\phi_{n}}$ (with $z$ and $\lambda$ fixed), then it holds for arbitrary $\phi$ in $L^{\infty}\left(m_{a}\right)$. This completes the proof of the theorem.

\section{SPECTRAL ANALysis OF TOEPLitz OPERATORS}

There are well-developed techniques for investigating the nature of the spectral resolution $A=\int t d E_{t}$ of a bounded selfadjoint operator $A$ on a Hilbert space $\mathscr{H}$ using, for $k$ in $\mathscr{H}$, the resolvent form

$$
\left\langle(A-\lambda)^{-1} k, k\right\rangle=\int \frac{1}{t-\lambda} d\left\|E_{t} k\right\|^{2}, \quad \operatorname{Im} \lambda \neq 0 .
$$


This form is a holomorphic function in the upper half-plane $\operatorname{Im} \lambda>0$ having positive definite imaginary part, consequently, one has a representation

$$
\Phi_{k}(\lambda)=1+\int \frac{1}{t-\lambda} d\left\|E_{t} k\right\|^{2}=\exp \left(\frac{1}{\pi} \int \frac{1}{t-\lambda} \delta_{k}(t) d t\right),
$$

where $\delta_{k}$ is a compactly supported measurable function satisfying $0 \leq \delta_{k} \leq 1$. Connections between the "phase shift" $\delta_{k}$ and the measure $d\left\|E_{t} k\right\|^{2}$ have been described in Aronszajn and Donoghue [2]. (See also, Donoghue [5].) We recall some of these connections.

I. Suppose that on an interval $(c, d)$ in $\mathbf{R}$ the function $\delta_{k}$, which is given a.e. with respect to Lebesgue measure by

$$
\delta_{k}(x)=\lim _{y \downarrow 0} \frac{1}{\pi} \operatorname{Im} \log \Phi_{k}(x+i y),
$$

satisfies

$$
\omega(k, c, d)=\operatorname{ess} \sup \left\{\left|\delta_{k}(t)-\delta_{k}(s)\right|: t, s \in(c, d)\right\}<1,
$$

then the measure $d\left\|E_{t} k\right\|^{2}$ is absolutely continuous on $(c, d)$.

II. The set $S_{k}$ defined by

$$
S_{k}=\left\{x: \lim _{y \downarrow 0} \operatorname{Im} \Phi_{k}(x+i y)=\infty\right\}
$$

is a support set for the singular component of the measure $d\left\|E_{t} k\right\|^{2}$.

III. The absolutely continuous component of the spectral measure $d\left\|E_{t} k\right\|^{2}$ is given by

$$
\frac{d\left\|E_{t} k\right\|^{2}}{d t}=\lim _{y \downarrow 0} \frac{1}{2 \pi i}\left(\left\langle(A-(t+i y))^{-1} k, k\right\rangle-\left\langle(A-(t-i y))^{-1} k, k\right\rangle\right) .
$$

Let us return to the resolvent form for the selfadjoint Toeplitz operator $T_{\phi}$ as given by Theorem 1. An argument similar to the one in Rosenblum [11, Lemma 1] can be used to establish the integrability of the function $\log |\phi-x|$, for a.e. $x$ in $\mathbf{R}$, with respect to the measure $m_{a}$ or any of the signed measures $* d \omega_{j}, j=1, \ldots, g$.

The following proposition is an immediate consequence of Theorem 1 and III.

Proposition 2. Let $\phi$ be a real-valued element in $L^{\infty}\left(m_{a}\right)$ and for $z$ fixed in $D$ let $k_{z}$ denote the reproducing kernel in $H^{2}\left(m_{a}\right)$. If $T_{\phi}=\int t d E_{t}$ is the spectral resolution of the selfadjoint Toeplitz operator $T_{\phi}$, then for almost all $t$ in $\mathbf{R}$

$$
\frac{d\left\|E_{t} k_{z}\right\|^{2}}{d t}=C(z) e^{-\int_{\partial D} \log |\phi-t| d m_{z}} \frac{1}{2 \pi} \operatorname{Im}\left(\psi(t) e^{i \pi \int_{A_{t}} d m_{z}}\right),
$$

where $A_{t}=\{p \in \partial D: \phi(p)<t\}$ and

$$
\psi(t)=\frac{\theta\left(\omega_{z}+t_{a}-(1 / 2 \pi) \int_{\partial D} \ln |\phi-t| d \vec{\nu}+(i / 2) \int_{A_{t}} d \vec{\nu}\right)}{\theta\left(t_{a}-(1 / 2 \pi) \int_{\partial D} \ln |\phi-t| d \vec{\nu}+(i / 2) \int_{A_{t}} d \vec{\nu}\right)},
$$

with $d \vec{\nu}=\frac{1}{2} * d \vec{w}$ a vector signed measure on $\partial D$. 
Remarks. (i) The function $\psi$ appearing in the above proposition is nonzero and finite a.e. on $\mathbf{R}$. This last remark can be seen as follows. Since $\theta$ is $\mathbf{Z}^{g}$ periodic, then for any $x$ in $\mathbf{R}^{g}$ the function

$$
\theta\left(x+\frac{1}{2 \pi i} \int_{\partial D} \log (\phi-\lambda) d \vec{w}\right)
$$

is a bounded holomorphic function in the upper half-plane $\operatorname{Im} \lambda>0$. Consequently, this function will have nonzero finite nontangential limiting values at almost every $t$ in $\mathbf{R}$. The function $\psi$ is the ratio of two nontangential limit functions of this form.

(ii) It can be that $d\left\|E_{t} k\right\|^{2} / d t$ is zero for almost every $t$. For example, if $\phi$ is constant on each component of $\partial D$, then $T_{\phi}$ will be purely singular. This follows from general principles (see, for example, Abrahamse [1]). The real numbers $t$ not in the range of $\phi$, where

$$
\theta\left(t_{a}+\frac{1}{2 \pi i} \int_{\partial D} \log (\phi-t) d \vec{w}\right)
$$

vanishes are the eigenvalues of $T_{\phi}$ [4]. Consequently, if $\phi$ is constant on each component of $\partial D$, then, for $t$ not an eigenvalue or in the range of $\phi$, $d\left\|E_{t} k_{z}\right\|^{2} / d t$ will be zero. This fact can be verified directly from the result in Proposition 2 by using the fact that for such a point $t$ the vector $(i / 2) \int_{A_{t}} d \vec{\nu}$ is a half-period.

Proposition 3. Let $\phi$ denote a real-valued element in $L^{\infty}\left(m_{a}\right)$ which is either a simple function or analytic. The singular continuous component of $T_{\phi}$ is trivial.

Proof. Assume first that the symbol $\phi$ is a simple function. In this case for $z$ fixed in $D$, the function appearing on the right side of equation (2.5) is continuable from the upper half-plane $\operatorname{Im} \lambda>0$ to the real axis minus the set of values of $\phi$ and having at most a countable set of poles at the zeros of

$$
h(\lambda)=\theta\left(t_{a}-\frac{1}{2 \pi i} \int_{\partial D} \log (\phi-\lambda) d \vec{w}\right) .
$$

The zeros of $h$ can only accumulate at the points in the range of $\phi$. One can conclude from property II of the resolvent form that the singular component of the measure $d\left\|E_{t} k_{z}\right\|^{2}$ is contained in the union of the zero set of $h$ and the values of $\phi$. Since the closed span of the reproducing kernels $k_{z}$, as $z$ varies over $D$, is all of $H^{2}\left(m_{a}\right)$, then the only possible singular component of $T_{\phi}$ is point spectra (which may or may not exist).

The proof for the case where the symbol $\phi$ is analytic on $\partial D$ is similar. In this case the right side of equation (2.5) can be continued onto the real axis minus the set of values $\lambda$ which correspond to branching of the multiple valued function $\lambda=\lambda(p)$ defined by $\phi(p)-\lambda=0$. These branching points are the only possible accumulation points of the zeros of the function $h$ defined in equation (3.1). The rest of the details are as above. This completes the proof of the proposition. 
In the doubly connected case we have the following complete result.

Proposition 4. Let $D$ be doubly connected and $\phi$ an arbitrary real-valued element in $L^{\infty}\left(m_{a}\right)$. The singular continuous component of the operator $T_{\phi}$ is trivial.

Proof. The only zero of the function $\theta$ in the Jacobian variety is at the halfperiod $[1 / 2+\tau / 2]$. The only way that the function $h$ defined in (3.1) can approach zero as $\lambda=t+i y$ approaches $t$ with $y \downarrow 0$ is if

$$
\left[\frac{1}{2} \int_{A_{t}} d \vec{w}\right]=\left[\frac{1}{2} \tau\right] .
$$

This condition can happen if and only if $M_{0} \leq t \leq m_{1}$ or $M_{1} \leq t \leq m_{0}$, where $M_{j}=\operatorname{ess} \sup \left\{\phi(p) \mid p \in b_{j}\right\}$ and $m_{j}=\operatorname{essinf}\left\{\phi(p) \mid p \in b_{j}\right\}, j=0,1$. It may be that $\left[M_{0}, m_{1}\right]$ or $\left[M_{1}, m_{0}\right]$ fail to be intervals, however, if they are intervals, then $h$ can have at most a countable number of zeros in these intervals accumulating only at the ends. On the complement of the union of $\left[M_{0}, m_{1}\right]$ and $\left[M_{1}, m_{0}\right]$, it follows from (2.5) and property $I$ of the resolvent form that $d\left\|E_{t} k_{z}\right\|^{2}$ is absolutely continuous. Thus $T_{\phi}$ is absolutely continuous off the union of $\left[M_{0}, m_{1}\right]$ and $\left[M_{1}, m_{0}\right]$ where it could have at most point spectra. The proof of the proposition is complete.

\section{REFERENCES}

1. M. B. Abrahamse, Toeplitz operators in multiply connected regions, Amer. J. Math. 96 (1974), 261-297.

2. N. Aronszajn and W. F. Donoghue, Jr., On exponential representations of analytic functions in the upper half-plane with positive imaginary part, J. Analyse Math. 5 (1956-57), 321-388.

3. K. F. Clancey, Representing measures on multiply connected planar domains, Illinois $\mathrm{J}$. Math. (to appear).

4. __ Toeplitz operators on multiply connected domains and theta functions, Operator Theory: Adv. Appl. 35 (1988), 311-355.

5. W. F. Donoghue, Jr., On the perturbation of spectra, Comm. Pure Appl. Math. 18 (1965), 559-579.

6. J. D. Fay, Theta functions on Riemann surfaces, Lecture Notes in Math., vol. 98, SpringerVerlag, New York, 1973.

7. R. S. Ismagilov, The spectrum of Toeplitz matrices, Dokl. Akad. Nauk SSSR 149 (1963), 769-772; English transl., Soviet Math. Dokl. 4 (1963), 462-465.

8. D. Mumford, Tata lectures on theta. I, II, Birkhauser-Verlag, Basel, 1983.

9. J. D. Pincus and J. Xia, Symmetric and self-adjoint Toeplitz operators on multiply connected plane domains, J. Funct. Anal. 59 (1984), 397-444.

10. M. Rosenblum, A concrete spectral theory for self-adjoint Toeplitz operators, Amer. J. Math. 87 (1965), 709-718.

11. _ Self-adjoint Toeplitz operators and associated orthonormal functions, Proc. Amer. Math. Soc. 13 (1962), 590-595.

12. E. I. Zverovich, Boundary value problems in the theory of analytic functions in Hölder classes on Riemann surfaces, Russian Math. Surveys 26 (1971), 117-192. 